\title{
Percepción materna de los signos, síntomas de alarma y creencias populares sobre el cuidado y las enfermedades neonatales en el departamento de emergencias pediátricas
}

\author{
Maternal understanding of warning signs and symptoms and \\ popular beliefs about newborn care and diseases in the \\ pediatric emergency department
}

Nadia Martínez¹, Mirta Mesquita ${ }^{1}$ Viviana Pavlicich¹

\section{RESUMEN}

Introducción: La percepción materna de los signos de alarma y las creencias populares influyen en el cuidado de los neonatos. El objetivo del presente trabajo fue describir la percepción materna de los signos de alarma y las creencias culturales relacionadas al cuidado del neonato. Material y Método: Estudio observacional descriptivo prospectivo. Previo consentimiento informado se realizó una entrevista, con preguntas abiertas y cerradas a madres de neonatos con nivel de prioridad de la atención II y III en el Departamento de emergencias pediátricas (DEP). Variables: Edad materna, escolaridad, procedencia, paridad, edad del neonato, antecedente de haber estado hospitalizado, motivo de la consulta, orientaciones sobre los cuidados neonatales, concepto de fiebre, signos de enfermedad grave y las llamadas enfermedades culturales. Los datos fueron procesados en SPSS v21 utilizando estadísticas descriptivas. Resultados: Se encuestó a 100 madres, la mayoría procedente del departamento Central, con estudios secundarios y más de la mitad era primíparas. El 63\% definió correctamente la fiebre. Solo el $25 \%$ recibió orientación sobre los cuidados del neonato por personal sanitario. El 90\% reconoció la fiebre como signo de alarma. El $25 \%$ manifestó creer en las llamadas enfermedades culturales. El 40\% de los neonatos usaba ombliguero, y el 51\% llevaba una cinta roja atada a la muñeca como medida de protección contra el mal. Conclusiones: La mayoría de las madres tuvo una adecuada percepción de al menos 2 signos de alarma Un cuarto de estas mujeres creía en las enfermedades culturales y más de la mitad de sus neonatos tenía amuletos contra el mal.

Palabras clave: Enfermedades culturales, cuidados neonatales, percepción materna, signos de alarma, urgencias pediátricas.

\begin{abstract}
Introduction: Maternal understanding of warning signs and symptoms and popular beliefs influence the care of newborns. The objective of this study was to describe the maternal understanding of warning signs and the cultural beliefs related to newborn care. Methods: This was a prospective, descriptive, observational study. After obtaining informed consent, an interview, using open and closed questions was performed with mothers of newborns presenting with priority levels of care II and III in the Pediatric Emergency Department (PED). Variables: Maternal age, education, prove-nance, parity, age of the newborn, history of having been hospitalized, chief complaint, guidance on newborn care, understanding of fever and signs of serious illness and so-called cultural diseases. The data was processed in SPSS v21 using descriptive statistics. Results: 100 mothers were surveyed, most of them from Central Department and with secondary-level education. More than half were primiparous. $63 \%$ correctly defined fever. Only 25\% received guidance from health personnel re-garding newborn care. $90 \%$ recognized fever as a warning sign. $25 \%$ said they believe in the so-called cultural diseases. $40 \%$ of neonates wore bellybands, and $51 \%$ wore a red ribbon tied to the wrist as a protective measure against evil. Conclusions: Most mothers had an adequate understan-ding of at least 2 warning signs. A quarter of these women believed in cultural diseases and more than half of their neonates had amulets against evil.
\end{abstract}

Keywords: Cultural diseases, neonatal care, maternal understanding, warning signs, pediatric emer-gencies.

\footnotetext{
${ }^{1}$ Hospital General Pediátrico Niños de Acosta Nu. San Lorenzo, Paraguay.

Correspondencia: Mirta Mesquita. Email:mirtanmr@gmail.com

Conflicto de Interés: Los autores declararan que no poseer conflicto de interés

Recibido: 18/04/2018. Aceptado: 27/04/2018
}

DOI: https://doi.org/10.31698/ped.45012018007 


\section{INTRODUCCIÓN}

La mortalidad neonatal constituye un grave problema de salud pública en América Latina y el Caribe (ALC). Las tasas de mortalidad infantil se han reducido en la región, sin embargo la mortalidad neonatal se ha modificado poco. ${ }^{(1)}$ Aunque las principales causas de mortalidad neonatal están bien definidas, (infecciones, asfixia, prematuridad y malformaciones congénitas), existen otros factores que contribuyen a una salud neonatal deficiente en países poco desarrollados, entre los que se encuentra las desigualdades a los accesos de salud, escasa atención por personal capacitado y salud materna precaria. $^{(2)}$

La salud y la supervivencia de los neonatos en el post parto, dependen en gran medida de la atención de las madres y/o cuidadoras. En países en vías de desarrollo tanto de Latinoamérica como de otras regiones, las creencias y costumbres populares, influyen en las decisiones que toman las madres en lo referente a los cuidados que deben tener los neonatos, así como en la percepción de la gravedad de los síntomas que presentan. Posiblemente la etapa neonatal sea donde mas tabúes existen sobre la interpretación de los signos y síntomas que presentan. ${ }^{(3)}$ En el departamento de emergencias pediátricas (DEP) del hospital donde se realizo el estudio, los neonatos constituyen el $2 \%$ de las consultas (unos 1300 neonatos al año). En el 48\% de los casos no requieren una atención urgente. ${ }^{(4)} \mathrm{El}$ objetivo de presente estudio fue describir la percepción materna de los signos de alarma o de riesgo de enfermedad grave y las creencias culturales relacionadas al cuidado de los recién nacidos que consultan en elDEP.

\section{MATERIAL Y MÉTODOS}

Estudio observacional descriptivo prospectivo de corte transverso. En el periodo comprendido entre octubre a diciembre del 2016, una de las investigadoras, previo consentimiento informado, realizó una entrevista con un cuestionario, con preguntas abiertas y cerradas a madres de neonatos con nivel de prioridad de la atención II y III, al finalizar la consulta en el DEP. El muestreo fue no probabilístico, a conveniencia de la entrevistadora de acuerdo al horario que disponía. El tamaño de la muestra calculado para poblaciones finitas fue de 96 participantes, con un intervalo de confianza al $95 \%$.

Se consideraron las variables, edad materna, escolaridad, procedencia, paridad, edad del neonato, antecedente de haber estado hospitalizado y el motivo de la consulta. Se pregunto a las madres si habían recibido orientación sobre los cuidados que requieren los recién nacidos y que concepto tenían de la fiebre. A partir de una lista de signos y síntomas banales y graves, se les pidió que manifestaran aquellos que consideraban como de riesgo de enfermedad grave. La lista se creó a partir de los motivos de consulta más frecuentes en la base de datos en la base del DEP. Además en la pregunta abierta se les pidió que indicaran la conducta que tomarían si notaban a sus recién nacidos con ictericia (preguntando como coloración amarilla de piel) y con lesiones en la piel (maculas, pústulas, signos inflamatorios). Se indagó además sobre las creencias y enfermedades culturales del recién nacido muy arraigadas en el país como el uso de cinta roja en la muñeca del neonato, razones para usar ombliguero, y las enfermedades de origen cultural guaraní como el Ojeo, (mal de ojo) Pya'ruru (distensión abdominal), Kambyryrujere (presencia de diarreas y vómitos). Se definió enfermedades culturales a aquellas que son conocidas por tradición de una generación a otra, y a las que se atribuye una causa, medios de diagnóstico y tratamiento con componentes sobrenaturales o mágicos. Los datos fueron procesados en SPSS v21 utilizando estadísticas descriptivas. El comité de ética de la institución aprobó el protocolo de estudio con consentimiento informado.

\section{RESULTADOS}

En el periodo de estudio se encuestó a 100 madres. Las mismas tenían un promedio de edad de 25,5 56,2 años la procedencia, paridad y escolaridad se observa en el la Tabla 1.

La mediana de edad de los neonatos fue de 18 días (mínimo de 3 máximo 28 días).El 17\% tenía antecedente de haber estado hospitalizado previa a la actual consulta. 
Los motivos de consulta se observa en la Tabla 2.

Tabla 1. Procedencia, por departamento, paridad y escolaridad de las madres de neonatos encuestadas $\mathrm{n}=100$

\begin{tabular}{|llcc|}
\hline & & N & \% \\
\hline Procedencia & Central & 88 & 88 \\
& Otros & 12 & 12 \\
\hline \multirow{2}{*}{ Escolaridad } & Primaria & 9 & 9 \\
& Secundaria & 73 & 73 \\
\hline & Terciaria & 18 & 18 \\
\hline Núm. de hijos & 1 & & \\
\hline & 2 a 5 & 51 & 51 \\
\hline Total & $>5$ & 48 & 48 \\
\hline
\end{tabular}

Tabla 2. Motivos de consulta de los neonatos de las madres encuestadas. $\mathrm{n}=100$

\begin{tabular}{|lcc}
\hline Motivo de consulta & N & \% \\
\hline Fiebre & 21 & 21 \\
\hline Congestión nasal & 10 & 10 \\
\hline Irritabilidad & 8 & 8 \\
\hline Lesiones de piel & 7 & 7 \\
\hline Vómitos & 6 & 6 \\
\hline Dificultad respiratoria & 6 & 6 \\
\hline Ictericia & 6 & 6 \\
\hline Control & 5 & 5 \\
\hline Remitidos de otro centro & 5 & 5 \\
\hline Sangrado umbilical & 5 & 5 \\
\hline Cólicos & 4 & 4 \\
\hline Estreñimiento & 4 & 4 \\
\hline Secreción ocular & 3 & 3 \\
\hline Rechazo pecho materno & 2 & 2 \\
\hline Diarrea & 2 & 2 \\
\hline Otros & 6 & 6 \\
\hline Total & $\mathbf{1 0 0}$ & $\mathbf{1 0 0}$ \\
\hline
\end{tabular}

Acerca del conocimiento sobre la temperatura de los neonatos el $26 \%$ de las madres consideraban a $\geq 37,5^{\circ}$ como temperatura elevada. El $63 \%$ definía a la fiebre como una temperatura mayor a $38^{\circ} \mathrm{C}$, el $5 \%$ mayor de $35^{\circ} \mathrm{C}$ y el $5 \%$ manifestaron desconocimiento acerca de los valores de la temperatura normal de sus neonatos.

Del total de la madres entrevistadas el 51\% (51/100) los neonatos eran producto del primer embarazo El $55 \%(55 / 100)$ de las madres refirieron que recibieron orientación sobre los cuidados de los recién nacidos durante el embarazo o en los primeros días del puerperio En cuanto a las personas que dieron la orientación se observa en la Tabla 3.
Tabla 3. Descripción de las personas que dieron orientación sobre los cuidados de un recién nacido a las madres que manifestaron haber recibidos información $\mathrm{n}=55$

\begin{tabular}{lcc}
\hline Persona que oriento & N & \% \\
\hline Madre de la entrevistada & 33 & 60 \\
Personal de salud (médico o enfermera) & 14 & 25,5 \\
Otros & 8 & 14,5 \\
Total & $\mathbf{5 5}$ & $\mathbf{1 0 0}$ \\
\hline
\end{tabular}

En relación a los síntomas que las madres consideraban de riesgo de enfermedades graves y por tanto debían buscar atención médica se describe en la Tabla 4.

Tabla 4. Signos y síntomas que las madres consideraron de riesgo de enfermedad

\begin{tabular}{lcc}
\hline Signos de alarma identificados por las madres. & N & $\mathbf{\%}$ \\
\hline Fiebre & 90 & 90 \\
Rechazo de la alimentación & 55 & 55 \\
\hline Secreción ocular & 53 & 53 \\
Falta de evacuación intestinal diaria & 39 & 39 \\
Presencia de una zona de la cabeza más blanda & 26 & 26 \\
que el resto (fontanela anterior) & & \\
No eructar después de cada alimentación & 21 & 21 \\
Despertarse cada 2 a 3 horas & 6 & 6 \\
Regurgitar & 5 & 5 \\
Defecar después de cada alimentación & 5 & 5 \\
\hline
\end{tabular}

Dos motivos importantes de consulta en el DEP del hospital, que se encuentran entre las 5 primeras causas de consulta en el DEP del hospital pediátrico (4) y que pueden eventualmente tener complicaciones graves, son la ictericia (formas de hiperbilirrubinemia severa) y las lesiones de piel (infecciones bacterianas severas).Las respuestas de las madres a la pregunta de cuál sería la conducta que tomarían si sus neonatos presentaran alguno de estos síntomas, fue: para la ictericia el $60 \%$ respondió que los llevaría a la consulta, el 37\%, le pondría al sol y el $2 \%$ le daría de beber té. Para las lesiones de piel el $89 \%$ refirió que le llevaría a consultar, el 10\% optaría por ponerle una crema y el $1 \%$ le bañaría más frecuentemente.

Se indagó sobre el conocimiento de enfermedades denominadas culturales basadas en creencias populares sobre los cuidados de los recién nacidos y que solo lo pueden diagnosticar y tratar los curanderos, mientras que los médicos universitarios los desconocen. Los resultados se muestran en la Figura 1. 


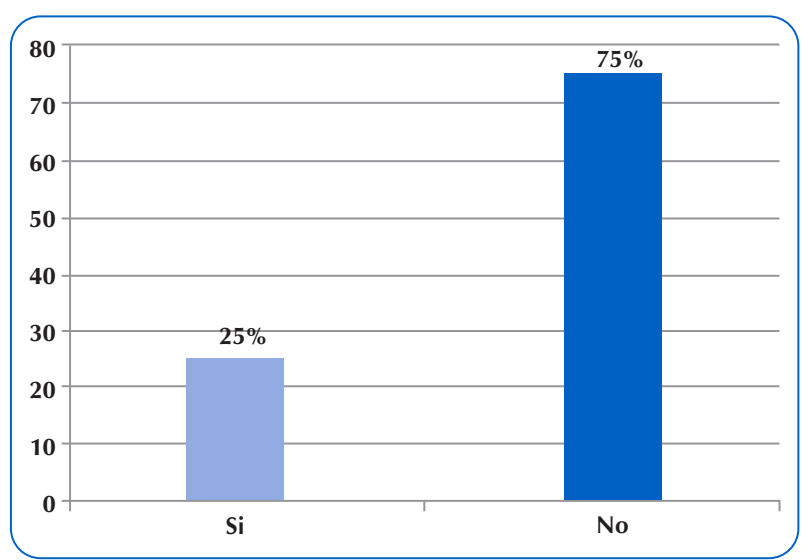

Figura. 1. Porcentaje de madres que manifestaron creer la existencia de enfermedades del recién nacido que conocen y tratan solo los curanderos. $n=100$

Los nombres populares y en idioma guaraní de las enfermedades citadas, se encuentran en la Tabla 5.

Tabla 5. Enfermedades culturales conocidas por las madres entrevistadas $\mathrm{n}=25$

\begin{tabular}{lcc}
\hline Enfermedades Culturales & N & \% \\
\hline Ojeo & 7 & 28 \\
Py'a ruru & 6 & 24 \\
Kambyryrujere & 4 & 16 \\
Combinación de las 3 & 8 & 32 \\
Total & $\mathbf{2 5}$ & $\mathbf{1 0 0}$ \\
\hline
\end{tabular}

Una práctica frecuente en el país es el uso de ombligueros en los recién nacidos. Se indago acerca de la misma al grupo de madres entrevistadas. El $40 \%$ manifestó que lo utilizan y por varias razones: el $62,5 \%$ (25/40) para evitar la hernia umbilical, el 20\% (8/40) calmar el dolor abdominal, el 7,5\%(3/40) prevenir la infección umbilical, el 7.5\%(3/40) para evitar roce umbilical del pañal y el $2.5 \%(1 / 40)$ para mejorar el sueño del neonato.

Otra de las tradiciones en el país es colocar una cinta roja alrededor de la muñeca del recién nacido. El $54 \%$ de los neonatos del estudio lo portaban. Al preguntar a sus madres cual era el significado del mismo, el 70\% respondió que como no estaban aun bautizados esto los protegía de los" malos espiritus,", el 12,9\% para evitar el "ojeo", el 5,5\% por recomendación de familiares. Otras razones fueron para evitar tenga enfermedades, pesadillas, y que no sea "cabezudo".

\section{DISCUSIÓN}

La mayoría de las madres percibió correctamente al menos dos signos de alarma muy importantes en el neonato como la fiebre y el rechazo alimentario. Estos resultados son similares a los encontrados en un estudio realizado en Nigeria, donde el $92 \%$ de las madres percibieron a la fiebre como un signo de alarma, seguido del rechazo de la alimentación en el $40 \%{ }^{(5)}$. Y muy superior a los resultados de Dongre AR y cols. quiénes entrevistaron a un grupo de madres que acudieron a un centro de salud en un distrito rural de la India, $67 \%$ de las cuales pudo identificar al menos 1 signo de alarma ${ }^{(6)}$. Sin embargo características físicas y fisiológicas propias del neonato como la presencia de la fontanela anterior la falta de evacuación diaria, regurgitación y la periodicidad de la alimentación fueron consideradas erróneamente como signos de alarma en casi la tercera parte de las madres encuestadas. Este hallazgo refleja la falta de información de las mismas sobre las características y los cuidados de los recién nacidos. Resultados similares se han encontrado en otros reportes sobre las características de las madres de los de los neonatos que consultan en un $\operatorname{DEP}^{(4,7,8)}$.

En la percepción materna, de los cuidados que debe tener un neonato, influyen numerosos factores como el nivel educativo de las mismas, entendido no solo como escolaridad sino también como la capacidad de internalizar valores y creencias que se encuentran fuertemente relacionados con la diversidad cultural. En un trabajo multicéntrico realizado en varios países del sur de Asia, con el objeto de evaluar la calidad de los cuidados del neonato, los resultados finales concluyeron que las creencias culturales y religiosas estaban entre los principales obstáculos en la búsqueda de la atención del recién nacido ${ }^{(9)}$.

Otros factores maternos que influyen en la búsqueda de atención médica, es la falta de experiencia y la escasa orientación sobre los cuidados del recién nacido durante el embarazo, que les genera mucha ansiedad e inseguridad a la hora de evaluar los síntomas del infante. ${ }^{(10)}$ Estas dos características la hemos encontrado en el grupo de madres participantes. Más de la mitad era primigestas y no había recibido orientación por el personal de salud sobre los cuidados del neonato. El nivel de 
educación de las madres, el acompañamiento de la pareja, y la información suministrada a la madre durante el embarazo fueron factores asociados a un mejor conocimiento de los signos de peligro de la salud neonatal, en un estudio realizado en Nigeria ${ }^{(11)}$ En Canadá Ahronhein SR y cols realizaron una entrevista a futuros padres con el objetivo de evaluar la actitud que tomarían ante la posibilidad de enfermedad de sus neonatos. No encontraron asociación entre la opción de buscar atención medica y características socio demográficas de los participantes. El estudio destaca que a pesar del acceso a la salud de alta calidad en Canadá se encontró brechas en el conocimiento del niño enfermo en una quinta parte de los futuros padres ${ }^{(12)}$. La mayoría de las madres encuestadas reconoció que la presencia de ictericia y lesiones en la piel del recién nacido como motivos para buscar atención médica, en concordancia con los resultados reportados por otros autores sobre el tema ${ }^{(13)}$. Sin embargo en Malasia, la mayoría de las mujeres encuestadas en un hospital, manifestó que colocaban a sus recién nacidos con ictericia, bajo el sol sin considerar la consulta médica previa ${ }^{(14)}$.

Las llamadas enfermedades culturales del neonato y lactante pequeño, en países poco desarrollados, son responsables de la consulta médica tardía. Comprende un grupo de signos y síntomas cuyas causas pueden ser enfermedades graves como la sepsis o meningitis y cuyo tratamiento, de acuerdo a la tradición popular, requiere participación de fuerzas sobrenaturales administradas por curanderos o chamanes a los que se recurre, antes que a la atención médica. ${ }^{(15)}$ En el presente estudio un cuarto de las mujeres participantes, la mayoría con estudios secundarios y de procedencia urbana, creía en la existencia de dichas enfermedades. En un estudio realizado en Pakistán el $45 \%$ de las 500 madres encuestadas creía que los métodos espirituales cumplían un papel preponderante en la sanación de las enfermedades ${ }^{(15)}$. En una región rural de Colombia un estudio cualitativo realizado con el objetivo de conocer la percepción de los signos de peligro de los neonatos, encontraron una buena concordancia entre grupos de madres y trabajadores de la salud. Sin embargo emergieron dos enfermedades culturales el "ojo" y el "espanto" como barreras para la atención adecuada y en tiempo del neonato enfermo ${ }^{(16)}$.

Otros cuidados neonatales basado en tradiciones y no en evidencias científicas demostrada, es el uso de ombliguero, paño arrollado al abdomen a nivel del cordón umbilical. Poco menos de la mitad de neonatos en el presente estudio lo utilizó. En algunos países africanos las madres utilizan sustancia como agua salada, polvo de casa o excrementos de lagartos como medidas de cuidado ${ }^{(17)}$.

Dentro de las creencias populares que amalgaman la tradición con fuerzas sobrenaturales como los malos espíritus, el "ojeo" entre otros, se encuentra la utilización de una cinta de color rojo alrededor de la muñeca del recién nacido. Mas de la mitad de los neonatos participantes portaban una cinta roja alrededor de la muñeca.

El presente estudio tiene la limitación de tratarse de un estudio cuantitativo, quizás los datos recogidos a partir de un enfoque cualitativo lo hubieran enriquecido. Sin embargo, lo consideramos muy útil como una instantánea que aporta datos sobre los cuidados y creencias maternas que se relacionan con la búsqueda de atención medica en el DEP, para un grupo muy vulnerable de pacientes como son los neonatos y puede constituirse en el antecedente de una investigación cualitativa que posteriormente pueda ser utilizada para orientar a las embarazadas y mejorar los cuidados del recién nacido.

\section{CONCLUSIONES}

La mayoría de las madres tuvo una adecuada percepción de al menos 2 signos de alarma muy importantes en el periodo neonatal (fiebre y rechazo de la alimentación). Se trataba de madres primerizas, con estudios secundarios de procedencia urbana, que recibieron orientación sobre los cuidados neonatales de algún familiar, y solo en pequeño porcentaje de parte del personal de salud. Un alto porcentaje las consultas fue por motivos relacionados a las características fisiológicas del neonato. Un cuarto de estas mujeres creía en las enfermedades culturales y más de la mitad de sus neonatos tenía amuletos que "espantan a malos espíritus" 


\section{REFERENCIAS}

1. Organización Panamericana de la Salud. Mortalidad materna y neonatal en ALC Organización y estrategias de reducción. Síntesis de situación y enfoque estratégico. Internet. 2011. Consultado el 30 de octubre del 2016. Recuperado a partir de: http://www.who.int/pmnch/acti vities/sientesis_situacionmortalidad:en_alc.pdf.

2. Organización Panamericana de la Salud. Reducción de la mortalidad y morbilidad neonatal en América Latina y el Caribe. Internet. 2007. Consultado el 1 de noviembre del 2016. Recuperado a partir de: http://www.publicaciones. ops.org.ar/publicaciones/saludAmbiental/RM/cdcofesa/ pdf/iaiepiRedMMNALyC.pdf

3. Awasthi S,Verma T,Agarwal M. Danger signs of neonatal illness : perceptions of caregivers and health workers in northern India. Bull World Health Oregan. 2006;84:819-26.

4. Mesquita M,Pavlicih V,Luaces C. El sistema español de triage en la evaluación de los neonatos en las urgencias pediátricas.Rev chil Pediatr 2017;88:107-112.

5. Kuganab _Lem R,Yidana A.Exploring women knowledge of newborn danger signs: a case of mothers with fewer tan five children .Public Health Res 2014;4: 195-202.

6. Dongre AR,Deshmukh PR, Garg BS. Percepcion of health care seeking about newborn danger signs among mothers in rural Wardha .Indian J Pediatr 2008;75:325-9.

7. Fernandez Ruiz C, Sainz de la Masa T, Curcoy Barcenilla AI Lasuen del Olmo N, Luaces Cubells C. Asistencia a neonatos en el servicio de urgencias del un hospital pediátrico terciario. AnPediatr (Barc). 2006; 65:123-8.

8. Perez Solis D, Pardo de la Vega J, Fernandez Gonzalez N, Ibañez Fernandez A, Prieto Espu nes S, Fanjul Fernandez L. Atencióna neonatos en una unidad de urgencias pediátricas. An Pediatr (Barc). 2003; 59:54.

9. Syed U,Khadka N,Khan A. Care-seeking practices in
South Asia:using formative research to design programs intervention to sabe newborn lives. J Perinatol 2008; 28 Suppl2S 9-13.

10. Kibaru EG, Otara AM, Knowledge of neonatal danger sings among mothers attending well baby clinic in Nakuru Central District Kenya; cross sectional descriptive study BMC Res Notes 2016;9: 481.

11. Ekwochi U,Ndu IK,Osuorah CD,Amadi OF, Okeke IB,Obioha E, et al. Knowledge of danger signs in newborns and health seeking practices of mothers and care givers in Enugu state,South east Nigeria.Ital J Pediatr 2015;41:18-22 12. Ahron heim SR,McGillivray D,Barbic S,Barbic D,Klam S,Brisebois Pet al.Expectant parent's understanding of the implications and management of fever inthe neonate,PLoS One 2015;8:10.

13. Ezeaka C,Ugwe R,Mukhtar YM,Olusanya B,Pattern and predictors of maternal care seeking practices for severe jaundice in Nigeria. A multi Centre survey.BMC Health Serv Res 2014;14:192-96.

14. Zang L,Hu P,Wang J,Zhang $\mathrm{M}$,Zhang KL,Hu B. Prenatal training improves new mother' understanding of jaundice.Med Sci Monit 2015;21: 1668-73

15. Mustafa G, Bashir N,Aslam ; Parenteral beliefs and practice of spiritual methods for the sick children at a tertiary hospital of Pakistan : a cross sectional questionnarie study.BMC Complement Altern Med 2016;16: 14.

16. Delgado-Noguera MF, Calvache-España JA, Tabares Trujillo RE, Del Cairo Silva C, Bedoya Idobro LM. Percepciones de signos de alarma en enfermedad neonatal , de los cuidadores y trabajadores de la salud en Guapi,Colombia. Rev Salud Publica 2007;9:32-52.

17. Waiswa P, Tomson Goran S,Pariyo GW. Poor newborn care practices - a populaion based survey in Eastern Uganda-BMC Pregnancy and Childbirth 2010;10:9. 